\title{
Distance Learning an steirischen Mittelschulen in Zeiten der COVID-19-Pandemie
}

\author{
Helene Weber ${ }^{1}$, Sandra Schön², Martin Ebner ${ }^{3}$
}

https://doi.org/10.53349/resource.2021.i16.a988

\section{Zusammenfassung}

Die COVID-19-Krise führte im März 2020 zur Schließung des gesamten österreichischen Schulbetriebs, was zur Folge hatte, dass Präsenzunterricht durch Distance Learning ersetzt werden musste. Der Beitrag stellt die Ergebnisse einer Master-Thesis vor, die das Ziel verfolgte, die technische und organisatorische Umsetzung von Distance Learning während der COVID-19-Krise an den steirischen Mittelschulen im Sommersemester 2020 zu untersuchen. An der schriftlichen Befragung nahmen 69 Direktor*innen, dies entspricht $42 \%$ der steirischen Mittelschulleitungen, teil. Der Beitrag beschreibt die schulischen Anordnungen, die Implementierung der Schulinitiativen und das Arrangement der Tools für Lern- und Lehrszenarien. Die Ergebnisse der Befragung zeigen, dass mehr als die Hälfte der steirischen Mittelschulen im Beobachtungszeitraum im Distance Learning keinem extra dafür erstellten Konzept für dessen Durchführung folgten. Videokonferenzen wurden von vielen Schulleitungen angeordnet und E-Mails stellten das beliebteste Kommunikationsmittel dar. Online-Lern- und Arbeitsplattformen wurden vermehrt eingesetzt, aber auch auf das Bereitstellen analoger Lernmaterialien wurde großer Wert gelegt.

Schlüsselwörter:

Distance Learning

Steirische Mittelschulen

Schulleitung

\section{Einleitung}

Durch die COVID-19-Pandemie wurde eine gesellschaftliche Krise ausgelöst, die sich auf beinahe alle Bereiche auswirkte (Huber et al., 2020, S. 15). Im Frühling 2020, ab dem 18. März, wurden auch in Österreich Schulschließungen angeordnet und der reguläre Präsenzunterricht in der traditionellen Form wurde untersagt (Faßmann, 2020, S. 1). Es wurden entsprechende Maßnahmen gesetzt, um den Unterricht als Distance Learning fortsetzen zu können (Tengler et al., 2020, S. 3); die Schulen standen nur noch für die Betreuung von Schüler*innen, deren Eltern in systemrelevanten Berufen arbeiteten bzw. alleinerziehend waren, offen (Tengler et al., 2020, S. 3; BMBWF, 2020a, Abs. 3). Zudem gab es strenge Hygienevorschriften und eine Einschränkung der sozialen Kontakte an den Schulstandorten (BMBWF, 2020a, Abs. 8). Mit diesen, in der jüngeren Geschichte einmaligen Maßnahmen hatte wohl niemand gerechnet und die Vorbereitungszeit war denkbar kurz. Es gab etliche praktische Probleme; kreative Lösungen an den Schulen sowie seitens der Lehrkräfte, der Schulverwaltung und der Eltern waren erforderlich (Wrase, 2020, S. 105-106). Der Stellenwert von Bildung, das Ansehen von Schulen und die Wertschätzung der Leistungen von Lehrer*innen sind durch die Krise aus Sicht des Bundesministers dabei wieder gestiegen (Faßmann et al., 2020, Abs. 7).

Während der Schulschließungen war die Schulleitung gemeinsam mit den Lehrkräften für die Organisation der analogen oder digitalen vertiefenden Übungsmaterialien verantwortlich und musste sich darum kümmern, dass sich auf den Lernplattformen stets Aufgaben befanden und auf Unklarheiten auf Seite der Lernenden online

\footnotetext{
${ }^{1}$ E-Mail: helene.weber@hotmail.com

2 TU Graz, 8010 Graz, Münzgrabenstraße 36/I

E-Mail: sandra.schoen@tugraz.at

${ }^{3}$ TU Graz, 8010 Graz, Münzgrabenstraße 36/I

E-Mail: $\underline{\text { martin.ebner@tugraz.at }}$
} 
reagiert wurde (Faßmann, 2020, S. 2). Die Schulen waren schulautonom für die Inhalte der Lernmaterialien zuständig, alle Klassen sollten jedoch Übungshefte für den Zeitraum bis zu den Osterferien erhalten, welche von den Schüler*innen bearbeitet werden mussten. Während dieser Phase musste sowohl der Kommunikationsprozess zwischen der Schule und den Erziehungsberechtigten als auch die Beantwortung von Fragen der Lernenden über neue Kommunikationskanäle erfolgen. Die Durchführung von (Über-)Prüfungen entfiel während dieses Zeitraums (BMBWF, 2020a, Abs. 4-11).

Nach den Osterferien wurde das Vermitteln von neuem Lernstoff via Distance Learning in allen Schulstufen und Unterrichtsgegenständen der Mittelschulen schulautonom genehmigt und auch ausgeführt. Schulleiter*innen entschieden mit dem Kollegium über die Medien, die im Distance Learning eingesetzt wurden, ebenso mussten alternative Angebote für den Fall, dass keine technische Ausrüstung vorhanden war, erarbeitet werden. Die Schulleitung kümmerte sich während des Distance Learnings auch um die Organisation und Förderung der Unterstützung von Lehrer*innen (BMBWF, 2020c, S. 1-3). Lehrpersonen mussten Feedback auf erledigte Aufgaben und Übungen geben (Faßmann, 2020, S. 2), die Bearbeitung der übermittelten Lernmaterialien wurde in der Leistungsbeurteilung wie Mitarbeit und Hausübung berücksichtigt (BMBWF, 2020b, S. 1).

Für die Aktivierung des Schulsystems nach dem ersten Lockdown im Frühjahr 2020 hat das Ministerium einen Etappenplan für alle Bildungseinrichtungen entworfen, der die zeitlichen und organisatorischen Perspektiven aufzeigt. Die Rückkehr der schulpflichtigen Kinder erfolgte unter Einhaltung der Hygiene- und Schutzmaßnahmen am 18. Mai 2020 (BMBWF, 2020d, S. 1-2). Das Ziel des Schichtbetriebs und der Verdünnung war es, das Infektionsrisiko durch die Reduzierung der Anzahl der Personen, die sich gleichzeitig an den Schulen aufhalten, zu minimieren. Das Schichtsystem schrieb die Teilung von Klassen in zwei jeweils gleich große Gruppen bis Schulschluss vor (BMBWF, 2020e, S. 3). Die Schulen konnten autonom entscheiden, ob die Schüler*innen täglich abwechselnd in die Schule kommen oder in mehrtägigen Blöcken wechselweise unterrichtet werden. Stundenpläne mussten aufrecht bleiben, Nachmittagsunterricht entfiel (BMBWF, 2020d, S. 3).

Die durch die COVID-19-Krise hervorgerufene Schulschließung forderte das Überdenken der Struktur von schulischen Vorgehensweisen, um für die Entwicklung der Lernenden flexibel handeln zu können (Langner, 2020, S. 111). Dabei fanden digitale Tools für die Bewältigung schulischer Aufgaben vermehrt Anwendung (Gerhardts et al., 2020, S. 139). Die COVID-19-Krise sorgte für eine neue Wahrnehmung des digitalen Lernens (Eickelmann, 2020, S. 6). Aufgrund der Schulschließung am 18. März 2020 wurden Schulen in Österreich mit zahlreichen neuen Herausforderungen konfrontiert. Beim Bewältigen dieser Herausforderungen spielten Koordination und Organisation eine zentrale Rolle. Sie trugen wesentlich zum erfolgreichen Distance Learning bei (Tengler et al., 2020, S. 3-4). Der Schwerpunkt lag auf der Umsetzung des Distance Learnings im Bereich der Organisation, der technischen Implementierung und der Didaktik (Brandhofer et al., 2020, S. 2).

Um Erkenntnisse zu der technisch-organisatorischen Umsetzung und den damit verbundenen Erfahrungen mit Distance Learning an steirischen Mittelschulen zu erlagen, verfolgte die Master-Thesis von Weber (2021) die Forschungsfrage "Wie wurde die Realisierung von Distance Learning während der COVID-19-Krise an steirischen Mittelschulen gemanagt?". In diesem Beitrag werden die wesentlichen Antworten auf die folgenden Teilfragen vorgestellt:

- Welches Konzept verfolgten die einzelnen steirischen Schulen während der Schulschließung?

- Wie erfolgte der Kommunikationsweg zwischen allen Personen, die am Funktionieren des Schulalltags beteiligt sind, und den Schüler*innen wie auch Eltern bzw. Erziehungsberechtigten?

- Welche Medien und Tools kamen bei der Realisierung des Distance Learnings an steirischen Mittelschulen am häufigsten zum Einsatz und für welche Funktionen wurden diese eingesetzt? Wie wurde der Lehrkörper auf die Anwendung dieser digitalen Medien geschult?

- Wie wurden die Erstellung der Unterrichtsmaterialien und die Absprache über Lerninhalte angeordnet?

Die Antworten auf diese Fragen wurden Abschnitten, nämlich „Realisierung der Schulkonzepte“, „Eingesetzte Tools" und "Kollegium“, zugeordnet und sind im Abschnitt „Ergebnisse“ zu finden. Zunächst beschreiben wir im Folgenden das methodische Vorgehen bei der Befragung und der Auswertung, dann die zentralen Ergebnisse der Befragung. Diese werden anschließend diskutiert und abschließend wird ein Fazit der Einsichten gezogen. 


\section{Methodische Vorgangsweise}

Weil Schulleitungen mit allen Aspekten der COVID-19-Maßnahmen für alle Jahrgänge vertraut sind und einen Gesamtüberblick haben, wurden sie als Adressat*innen der Befragung ausgewählt. Vor der Befragung wurde die Genehmigung der Bildungsdirektion für das Forschungsvorhaben eingeholt. Danach erfolgte die Kontaktaufnahme mit 164 steirischen Mittelschulleiter*innen. Die Daten wurden anonym erfasst und ausschließlich für den Forschungszweck ausgewertet.

Der Fragebogen ist auf der Plattform Zenodo zugänglich ${ }^{4}$. Er enthält, um ein schnelles Beantworten zu ermöglichen, nachvollziehbare sowie wenige Arten von Antwortmöglichkeiten und Items. Insgesamt umfasst die Online-Befragung 58 Fragestellungen, die für das Vorhaben entwickelt wurden. Die Fragen wurden größtenteils im Multiple-Choice-Format dargestellt. Vier Fragen mussten mit der Eingabe einer Zahl beantwortet werden; sieben offene Fragen wurden gestellt.

Die Befragung startete am 24. September 2020 und endete am 16. Oktober 2020. Die Teilnehmer*innen konnten die teilweise fertiggestellte Umfrage zwischenspeichern und diese zu einem späteren Zeitpunkt beenden. Nach eineinhalb Wochen wurde zur Erhöhung der Rücklaufquote eine Erinnerungsmail an die steirischen Schulleiter*innen gesandt. Abbildung 1 bietet einen Überblick der zeitlichen Abfolge von der Erklärung zur Pandemie durch den Generaldirektor der Weltgesundheitsorganisation, der ersten Schulschließung und dem Zeitpunkt der Online-Befragung.

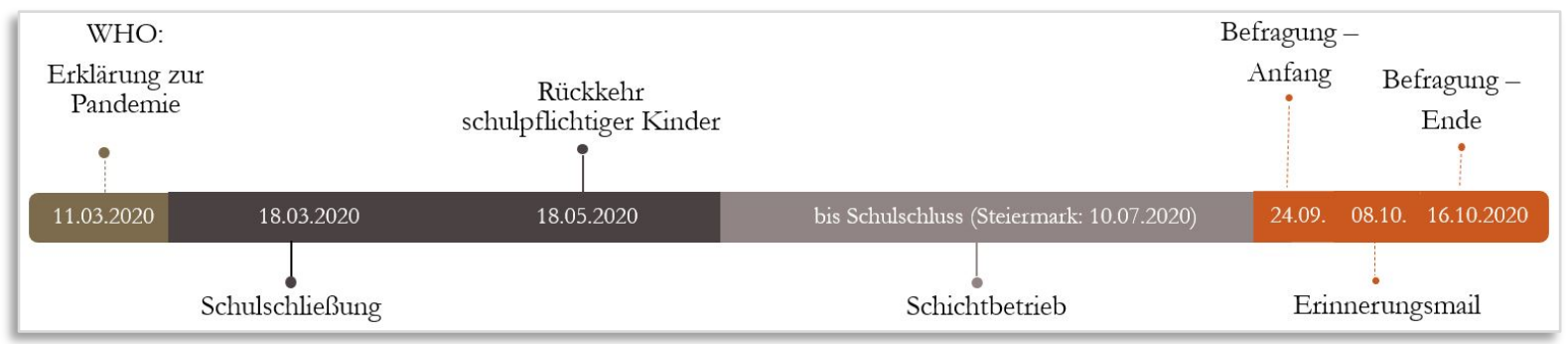

Abbildung 1: Zeitliche Abfolge der Entwicklungen an den österreichischen Schulen und der Befragung (nicht maßstabsgetreu)

Die anonyme Durchführung und die persönliche Kontaktaufnahme mit den 164 Mittelschulleiter*innen spiegeln sich in der Rücklaufquote wider: Die Online-Befragung wurde insgesamt von 85 steirischen Schulleiter*innen geöffnet; 16 Schulleitungen haben die Umfrage nur geöffnet und keine Fragen beantwortet. Neun Personen haben die Umfrage nicht vollständig abgeschlossen und die letzten Fragen der Online-Befragung nicht beantwortet. Die Antworten der abgeschlossenen Fragen wurden dennoch in den Ergebnissen berücksichtigt. Nach der Bereinigung der Daten standen für die Auswertung der Untersuchung insgesamt Antworten aus 69 Schulen zur Verfügung, was einer Rücklaufquote von $42 \%$ entspricht. Die Anzahl und der Anteil der Teilnehmer*innen für relevante und aussagekräftige Einschätzungen in Bezug auf die technischorganisatorische Umsetzung von Distance Learning an steirischen Mittelschulen sind damit sehr zufriedenstellend.

Die Auswertung der anonym erhobenen Daten erfolgte durch deskriptive statistische Verfahren. Die Auswertung und die Erstellung der Diagramme erfolgten mit Hilfe von Excel.

\section{Ergebnisse der Untersuchung}

Im Folgenden werden die Ergebnisse der Befragung präsentiert. Während als erstes die Darstellung der Mittelschulkonzepte und die Auflistung der eingesetzten Tools, die sowohl die Perspektive der Kommunikationsformen mit den Lernenden, den Erziehungsberechtigten und dem Kollegium beleuchten als auch die Kollaborationsformen analysieren, erfolgt, wird danach auf das Kollegium im Allgemeinen eingegangen. Anschließend werden die Antworten zur zukünftigen Bedeutung digitaler Medien vorgestellt und Anliegen, die

\footnotetext{
${ }^{4}$ Weber, H. (2021). Fragebogen für Distance Learning an steirischen Mittelschulen. URL: https://doi.org/10.5281/zenodo.5095375
} 
die steirischen Mittelschulen in Bezug auf Digitalisierung an die Regierung und an das Bildungsministerium haben, erläutert.

Die Rücklaufquote beträgt bei insgesamt 164 angeschriebenen steirischen Schulleiter*innen zufriedenstellende $42 \%$ ( $n=69$ ). Keine der Fragestellungen wurde von allen 69 Schulleitungen beantwortet, aus diesem Grund variiert die Zahl für $n$ stets. Die angeführte Teilnehmeranzahl bezieht sich im folgenden Kapitel auf die Anzahl der Schulleiter*innen, die die jeweils angeführte Fragestellung bzw. Zustimmungsmöglichkeit beantwortet haben. Die statistischen Kenngrößen ergeben sich aus der Berechnung der codierten Umfrageantworten und sind für die Interpretation der Forschungsergebnisse relevant. Bei der Auswertung wurde „trifft nicht zu“ mit dem numerischen Wert „1“, „trifft eher nicht zu“ mit „2", ,trifft eher zu“ mit „,3“ und „trifft zu“ mit „4“ codiert. Wegen der einfacheren Lesbarkeit sind die statistischen Kenngrößen abgekürzt (M=Mittewert, MD=Median, s=Standardabweichung).

\subsection{Vorgehen und Umsetzungen von Distance Learning und dem Einsatz digitaler Medien vor und während der ersten Schulschließung}

Zentral waren die Frage nach den bestehenden Konzepten für den Einsatz digitaler Medien vor der ersten Schulschließung, vorhandene Medienkonzepte sowie die Konzepte und Strategien der Schulen während der Schulschließung, d. h. Aufgabenverteilungen, digitaler Live-Unterricht, Kontaktaufnahme mit Erziehungsberechtigten, innerschulische Online-Fortbildungen, fachlicher Diskurs im Kollegium und schulinterne Kooperation sowie Administration und Verleihung bzw. Verschenken von Geräten. In Abbildung 2 werden die Einschätzungen der Schulleitungen zu entsprechenden Aussagen dargestellt.

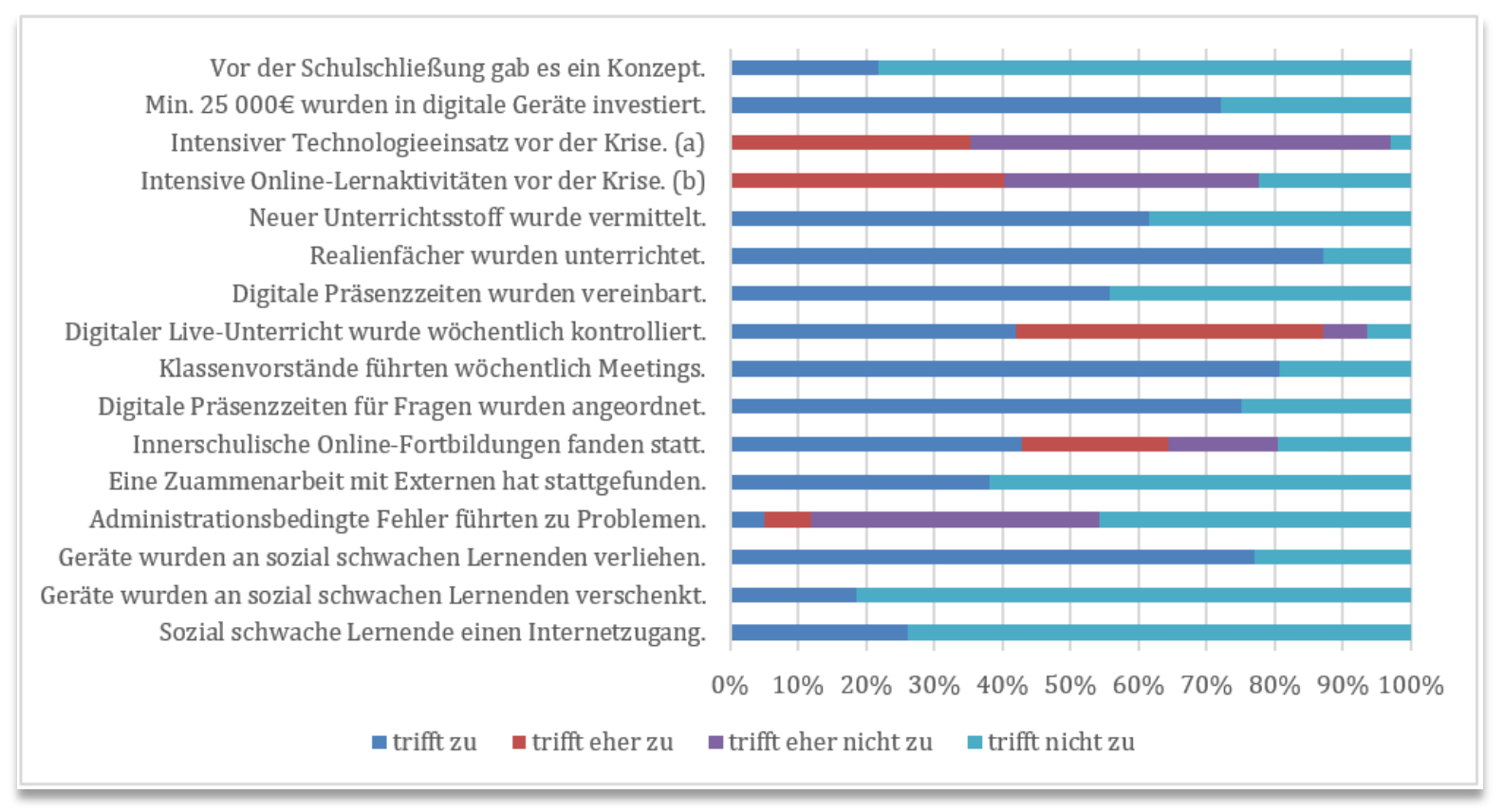

Abbildung 2: Umsetzungen und Anordnungen im Überblick. Ergebnisse der Befragung von 69 Schulleitungen an steirischen Mittelschulen im Oktober 2020.

Anmerkung: Die Originalitems lauten (a) „Vor der Schulschließung wurden mehrmals wöchentlich digitale Technologien im Unterricht eingesetzt“, (b) „Vor der Schulschließung wurden mehrmals wöchentlich Online-Lernaktivitäten (Lern- und Kommunikationsplattformen) durchgeführt“. Diese wurden hier aus Darstellungsgründen gekürzt.

Die Befragung ergab, dass $78 \%$ aller befragten Schulen $(n=64)$ vor den Schulschließungen kein Konzept gehabt hatten, das die Versorgung der Schüler*innen mit Lernangeboten für die Zeit einer Schulschließung sicherstellt. Nur $22 \%$ (14 Personen) der Teilnehmer*innen verfügten demnach über ein Konzept ( $M=1 ; M D=1$; $\mathrm{s}=0,413) .36$ ( $54 \%$ ) von 67 Schulleitungen gaben an, dass der Einsatz digitaler Medien zentral im Schulprogramm und Medienkonzept verankert ist und auch breite Anwendung findet. $42 \%$ der Schulleitungen sind der Meinung, digitale Medien zentral im Schulprogramm und Medienkonzept verankert zu haben und diese teilweise anzuwenden. Nur $5 \%$ (3 Schulen) gaben an, digitale Medien nicht oder nur randständig im Schulprogramm 
verankert zu haben. Außerdem haben 44 (72 \%) von 61 Schulen in den letzten zehn Jahren mindestens $25.000 €$ in digitale Geräte investiert, um einen modernen und digitalen Unterricht zu ermöglichen ( $M=2 ; M D=2 ; s=0,448)$.

Obwohl mehr als die Hälfte der befragten Schulen digitale Medien zentral im Schulprogramm berücksichtigen und diese auch breite Anwendung finden und fast zwei Drittel der Befragten viel Geld in digitale Geräte investiert hatten, hat keine der befragten steirischen Schulen $(n=68 ; M=2 ; M D=2 ; s=0,527)$ vor der Schulschließung mehrmals wöchentlich digitale Technologien im Unterricht eingesetzt oder mehrmals wöchentlich OnlineLernaktivitäten (auf Lern- und Kommunikationsplattformen) durchgeführt ( $n=67 ; M=2 ; M D=2 ; s=0,771$ ). $35 \%$ der Schulen gaben jedoch an, digitale Technologien vor der Schulschließung eher mehrmals wöchentlich im Unterricht eingesetzt zu haben und $40 \%$ haben vor der Schulschließung eher mehrmals in der Woche OnlineLernaktivitäten durchgeführt (siehe Abbildung 3).

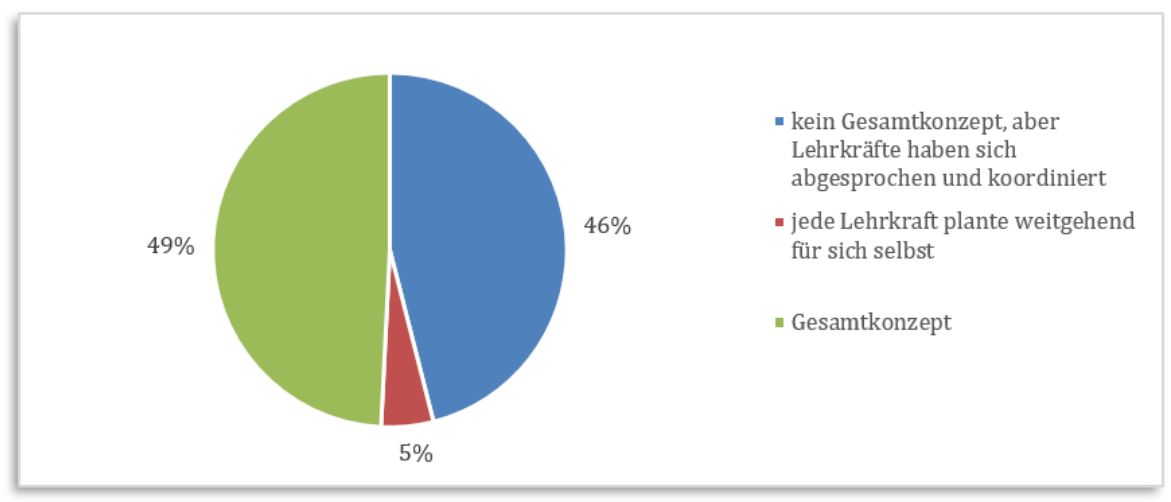

Abbildung 3: Gesamtkonzept der Schulen.

Ergebnisse der Befragung von 69 Schulleitungen an steirischen Mittelschulen im Oktober 2020.

Fast die Hälfte der befragten Direktor*innen $(n=63)$ gab an, während des Distance Learnings über ein Gesamtkonzept, das die Versorgung von Schüler*innen mit Lernangeboten für die Zeit der Schulschließung und die besondere Situation der Beschulung regelte, verfügt zu haben. $46 \%$ hatten zwar kein Gesamtkonzept, aber die Lehrkräfte der Schule haben sich abgesprochen und koordiniert, nur drei Schulen gaben an, dass jede Lehrkraft weitgehend für sich selbst plante $(M=2 ; M D=2 ; s=0,585)$.

Nach den Osterferien durfte in allen Schulstufen und Unterrichtsgegenständen der Mittelschulen schulautonom entschieden werden, ob neuer Lehrstoff durchgenommen wird. $62 \%$ der Schulen ( $n=52$ ) nannten die Anordnung der Vermittlung von neuem Unterrichtsstoff. Die restlichen 20 Schulen (38\%) fokussierten sich während dieses Zeitraums auf das Wiederholen von bereits unterrichteten Inhalten ( $M=2 ; M D=2 ; s=0,487)$. Auf die Zustimmung, ob die Schule das Unterrichten von Realienfächer während der Zeit des Distance Learnings angeordnet hatte, antworteten $87 \%(n=54)$ mit „trifft zu“. Nur $13 \%$ der Schulen gaben an, keine Anordnung für das Unterrichten von Nebengegenständen ausgesprochen zu haben ( $M=2 ; M D=2 ; s=0,336)$.

$56 \%$ der befragten Direktor*innen $(n=61)$ antworteten, Lehrer*innen mussten mit ihren Lernenden digitale Präsenzzeiten für Videokonferenzen vereinbaren. $44 \%$ (27 Schulen) verneinten diese Aussage ( $M=2 ; M D=2$; $s=0,497$ ). Von den $56 \%$ (34 Schulen), die der Aussage zustimmten, beantworteten 31 Direktor*innen die Aussage nach der mehrmaligen wöchentlichen Kontrolle des digitalen Live-Unterrichts. $42 \%$ antworteten, dass das Kontrollieren zutrifft, und $45 \%$, dass dies eher zutrifft. $6 \%$ antworteten jeweils mit "trifft nicht zu“ und ",trifft eher nicht $z u^{\prime \prime}$ ( $\left.n=31 ; M=3 ; M D=3 ; s=0,831\right)$.

Weiters wurde der Umfang der Anordnung des digitalen Live-Unterrichts pro Klasse/Gruppe von den $56 \%$, die der Aussage „Lehrer*innen mussten mit ihren Lernenden digitale Präsenzzeiten für Videokonferenzen vereinbaren" zustimmten, ausgewertet. 16 (52\%) der 31 befragten Schulleitungen antworteten, Videokonferenzen wurden zwei- bis dreimal pro Woche angeordnet. 26 \% führten einmal wöchentlich digitalen Live-Unterricht durch und sechs Schulen (19\%) bestanden darauf, dass täglich Videounterricht stattfand. Nur eine Schule gab an, dass Live-Unterricht alle zwei Wochen angeordnet wurde ( $n=31 ; M=4 ; M D=4 ; s=0,751)$.

Bei der Untersuchung wurde im Zusammenhang mit dem Umfang digitaler Live-Unterrichtseinheiten auch Bezug auf wöchentliche Meetings der Klassenvorständ*innen mit den Schüler*innen genommen. $81 \%$ der Schulleitungen $(n=31)$ gaben an, dass die Klassenvorständ*innen mindestens einmal in der Woche online eine Besprechung mit allen Schüler*innen der Klasse führen mussten, um sich über das Wohlbefinden und die 
aktuelle Situation auszutauschen $(M=2 ; M D=2 ; s=0,395)$. Außerdem ordneten drei Viertel der Schulleitungen $(n=32)$ digitale Präsenzzeiten für individuelle Fragen zu den Lerninhalten an $(M=2 ; M D=2 ; s=0,433)$.

Genauso wichtig wie die Anordnung der Umsetzung von Videokonferenzen während der Schulschließung ist die Art und Weise der Aufgabenverteilung, die an den steirischen Mittelschulen gewählt wurde, und wie die Rückmeldung dieser erfolgte. Die meisten Schulleitungen $(n=62)$ bevorzugten es, Aufgaben in Form von Wochenplänen oder in Kombination von Wochen- und Tagesplänen auszugeben ( $M=3 ; M D=3 ; s=1,428)$. 25 steirische Schulen gaben an, Aufgaben als Wochen- und Tagespläne verteilt zu haben, 22 weitere Schulen fokussierten sich nur auf die Ausgabe von Wochenpläne. Acht Direktor*innen gaben an, dass die Lehrer*innen sowohl Wochen- als auch Stundenpläne verteilten. Während nur zwei Schulleitungen Tagespläne anordneten, erfolgte die Aufgabenverteilung an einer Schule unsystematisch. Auf die Frage, wie häufig Lehrkräfte den Schüler*innen Rückmeldungen auf Aufgabenstellungen und Hausübungen geben mussten, antworteten $58 \%$ $(n=62)$ mit immer und $37 \%$ mit oft. Nur an $5 \%$ der befragten Schulen wurden Arbeitsaufträge gelegentlich rückgemeldet $(M=1 ; \quad M D=1 ; s=0,588)$. Die Schulen bevorzugten somit die Aufgabenverteilung durch Wochenpläne sowie Wochen- und Tagespläne und legten großen Wert auf regelmäßige Rückmeldungen.

Auch die Informationsübermittlung an die Eltern und Erziehungsberechtigten war während der Schulschließungen wichtig. $60 \%$ der 58 Direktor*innen gaben an, dass Informationen über Neuigkeiten wöchentlich erfolgten. $29 \%$ der Schulleitungen ordneten den Kontakt mit den Eltern und Erziehungsberechtigten mehrmals wöchentlich an. Fünf Schulleitungen hielten es für angemessen, die Eltern alle zwei Wochen zu kontaktieren, und an einer Schule erfolgte eine regelmäßige Kontaktaufnahme mit den Erziehungsberechtigten ( $n=58 ; M=3 ; M D=3 ; s=0,625)$.

Auch die Durchführung von innerschulischen Online-Fortbildungen zu den von der Schulleitung angeordneten Tools stellte für 24 von 56 Schulen bei der Umsetzung des Distance Learnings eine besondere Relevanz dar. $43 \%$ aller befragten Schulleitungen war es wichtig, dass den Kolleg*innen durch OnlineFortbildungen ein erleichterter Einsatz des jeweiligen Tools ermöglicht wird. $20 \%$ verzichteten auf innerschulische Fortbildungen, $16 \%$ der Schulen setzten eher keine Fortbildungen um und $21 \%$ war es eher wichtig, innerschulische Online-Fortbildungen für einen erleichterten Einsatz mit digitalen Medien zu ermöglichen.

52 Schulleitungen beschrieben ihr Konzept zum Erwerb von Kompetenzen der Kolleg*innen im Bereich der Anwendung des jeweiligen Tools. Mehrere Schulen gaben hier auch Mehrfachantworten. Am häufigsten nannten die Schulleitungen ( $n=52$ ) den Aspekt der gegenseitigen Unterstützung. $48 \%$ beschrieben das Aneignen von Kompetenzen durch Hilfestellungen und gegenseitigen Austausch im Kollegium. Es wurde versucht, Lösungen im Team zu finden und stets miteinander in Kontakt zu treten. $46 \%$ aller Schulleitungen äußerten, dass sich die Lehrkräfte Kompetenzen in Eigenregie aneigneten. Beim Selbststudium spielten Tutorials auf YouTube sowie auf Lernplattformen, von der Schule bzw. vom Kollegium aufgezeichnete Erklärungsvideos und Learning by Doing eine westliche Rolle. $40 \%$ der Schulen führten schulinterne Fortbildungen durch. Zur Aneignung digitaler Kompetenzen wurden Videokonferenzen und Online-Schulungen geführt, Weiterbildungen in Kleingruppen an der Schule abgehalten und das Fortbildungsangebot der Pädagogischen Hochschule Steiermark angenommen.

Zusätzlich wurde bei der Untersuchung der unterschiedlichen Konzepte auch das Ausmaß der fachlichen Diskurse im Kollegium und der schulinternen Kooperation zur Genese der Schul- und Unterrichtstrategie in Bezug auf digitale Medien erforscht. Aus den Antworten ging hervor, dass 26 Schulen $(n=60)$ regelmäßig fachliche Diskurse im Kollegium und eine schulinterne Kooperation führten. 13 Schulen setzten diese einmal in der Woche um und sieben Mittelschulen entschlossen sich dazu, alle zwei Wochen darüber zu sprechen. Sechs Schulen führten während der COVID-19-Krise mehrmals wöchentlich Gespräche über die Entwicklung einer Schul- und Unterrichtstrategie. Acht weitere Bildungseinrichtungen entschlossen sich, seltener als alle zwei Wochen darüber zu sprechen ( $M=2 ; M D=2 ; s=1,464)$.

Den Schulen waren der regelmäßige bzw. wöchentliche Austausch im Kollegium und die schulinterne Kooperation sehr wichtig, überraschenderweise jedoch nicht die Verankerung von Kooperationen in Bezug auf digitale Medien mit Externen. Während des Distance Learnings haben nur $38 \%$ der befragten Schulen $(n=55)$ Externe herangezogen. $62 \%$ aller steirischen Schulen versuchten, die Verankerung von digitalen Medien schulintern zu lösen ( $M=1 ; M D=1 ; s=0,486)$.

Da die Untersuchung zum Ergebnis gekommen ist, dass 62 \% der Schulen keine Externen für Kooperationen in Bezug auf digitale Medien herangezogen haben, ist die Anzahl der Personen, die an der Administration und an dem Funktionieren des von der Schule gewählten Tools beteiligt waren, umso wichtiger. Bei der angegebenen Personenanzahl der 60 steirischen Mittelschulen beträgt die Spannweite 30, wobei die drei Werte neun, zwölf und 32 als Extremwerte zu bewerten sind. Insgesamt nannten 22 Schulen drei Personen, neun Schulen jeweils vier bzw. fünf Beteiligte, zwei Schulen jeweils sechs und sieben Personen, drei Schulleitungen eine Lehrkraft und 
zehn Schulen zwei Beteiligte, die für das Funktionieren und Administrieren des Tools zuständig waren ( $n=60$; $\mathrm{M}=4 ; \mathrm{MD}=3 ; \mathrm{s}=4,074)$.

Als sehr positiv erscheinen bei der Untersuchung in Verbindung mit der Organisation Rückmeldungen über fehlende Wartungen und administrationsbedingte Fehler an der Schule, die nur bei $5 \%$ der Schulen ( $n=59)$ zu Problemen führten. $46 \%$ der Schulleitungen gaben an, dass keine fehlenden Wartungen und auch keine administrationsbedingten Fehler vorhanden waren, zusätzlich waren $42 \%$ der Meinung, dass diese eher keine Probleme bei der Durchführung der Lehr- und Lernprozesse verursachten ( $M=2 ; M D=2 ; s=0,804)$.

$77 \%$ von 61 Schulen gaben an, Geräte wie Computer, Laptops oder Tablets an die Schüler*innen verliehen zu haben ( $n=61 ; M=2 ; M D=2 ; s=0,421$ ). Von diesen 47 Schulen beantworteten 46, wie viele Geräte ungefähr verliehen wurden. Durchschnittlich wurden 11 Geräte pro Schule ausgeborgt. Werden Ausreißer nicht berücksichtigt, ergibt sich der Median mit durchschnittlich 7,5 Geräten pro Schule ( $n=46 ; s=10,369)$.

Im Gegensatz dazu gaben $19 \%(n=59)$ an, sozial schwachen Schüler*innen Geräte wie Computer, Laptops oder Tablets geschenkt zu haben ( $M=1 ; M D=1 ; s=0,389)$. Jede dieser zehn Schulen überreichte durchschnittlich 3 Schüler*innen ein Gerät ( $n=10 ; M D=2,5 ; s=1,578)$.

Zusätzlich zur Anzahl der verliehenen und verschenkten Geräte wurde erforscht, ob sich Schulen bei sozial schwachen Schüler*innen um einen Internetzugang kümmerten. Die Untersuchung zeigt, dass nur $26 \%$ der Schulen ( $n=58$ ) einen Internetzugang zur Verfügung stellen konnten. Von diesen 15 Schulen beschrieben zehn, den Internetzugang bzw. die Geräte über Spenden finanziert zu haben.

\subsection{Eingesetzte Tools}

Dieser Abschnitt widmet sich den Tools, die von den steirischen Mittelschulen eingesetzt wurden. In diesem Abschnitt wird auf die eingesetzten digitalen Medien, die sowohl aus der Perspektive der Rückmeldungen, der Kommunikationswege, der angewendeten Online-Lern- und Arbeitsplattformen als auch aus der Sicht der Informationsvermittlungen beleuchtet werden, Bezug genommen. Die Antworten der Fragestellung hinsichtlich der Kommunikationswege zwischen dem Kollegium und der Schulleitung ( $n=66$ ) wird in Abbildung 4 dargestellt. Mehrfachantworten wurden ermöglicht, da häufig mehrere Kommunikationsmittel eingesetzt wurden.

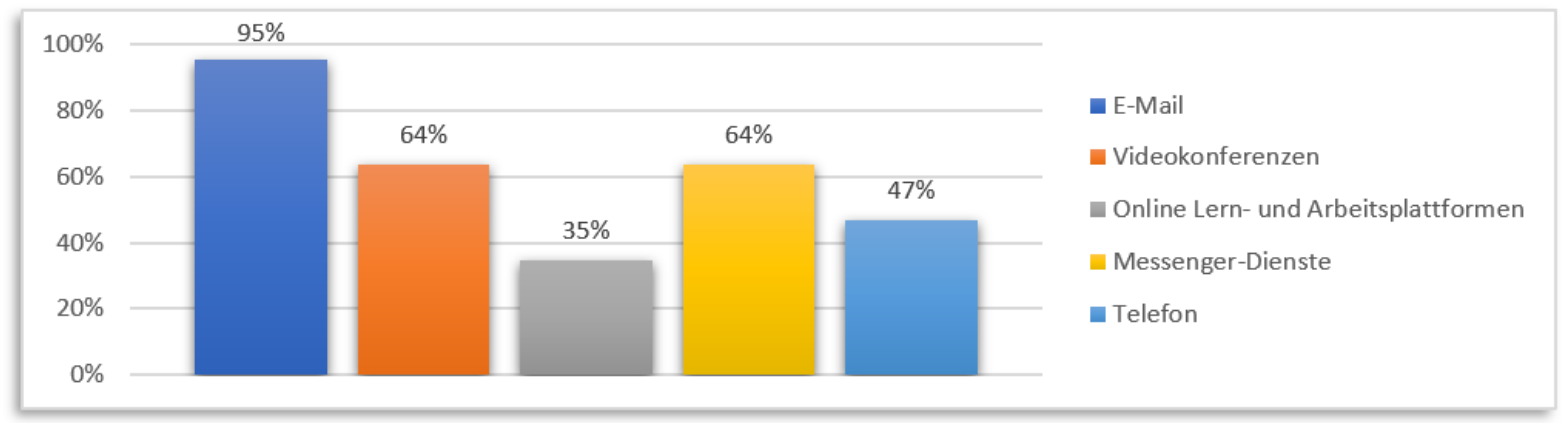

Abbildung 4: Kommunikation zwischen Kollegium und Direktion ( $n=66)$.

Ergebnisse der Befragung von 69 Schulleitungen an steirischen Mittelschulen im Oktober 2020.

Auffallend ist, dass $95 \%$ aller Befragten das Schreiben von E-Mails als Kommunikationsmittel bevorzugten. 64 \% nutzten Videokonferenzen wie MS Teams, Zoom etc. und Messenger-Dienste, um die Kommunikation aufrechtzuerhalten und Informationen auszutauschen. Fast die Hälfte (47\%) der Schulleitungen gab an, Informationen und Neuigkeiten telefonisch zu vermitteln. Nur $35 \%$ der Befragten tauschten Informationen mit dem Kollegium über Online-Lern- und Arbeitsplattformen aus. Zudem nannten zwei Schulen den Austausch vor Ort und eine Schulleitung gab an, Informationen und Neuigkeiten auch über EduPage ausgetauscht zu haben.

Während Online-Lern- und Arbeitsplattformen (Eduvidual, Google Classroom, MS Teams etc.) beim Informationsaustausch zwischen Direktion und Kollegium nur bei rund einem Drittel eine bedeutende Rolle einnahmen, waren diese für die Kommunikationswege mit den Lernenden äußerst wichtig - siehe Abbildung 5. 


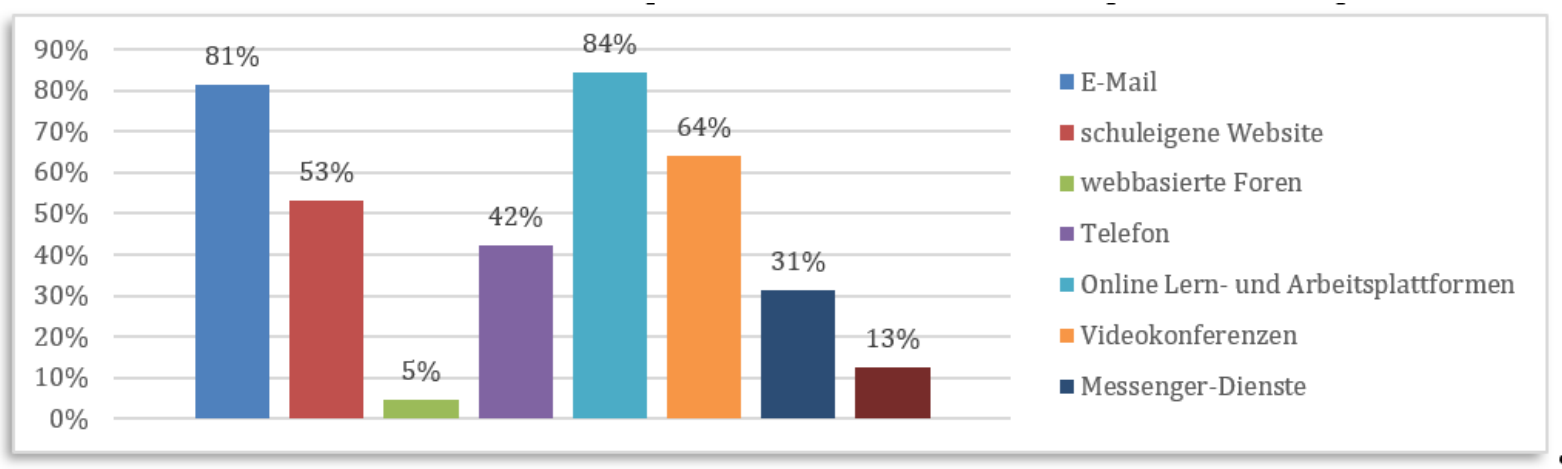

Abbildung 5: Kommunikationswege für Kontakt mit Schüler*innen ( $n=64)$. Ergebnisse der Befragung von 69 Schulleitungen an steirischen Mittelschulen im Oktober 2020.

$84 \%$ der befragten Schulen $(n=64)$ ordneten das Nutzen von Online-Lern- und Arbeitsplattformen an, um Schüler*innen zu kontaktieren und über Neuigkeiten zu informieren. Fast genauso wichtig war das Schreiben von E-Mails, das von 81 \% der Schulleitungen als Kommunikationsweg gewählt wurde. 64 \% der Schulen führten Videokonferenzen (MS Teams, Zoom etc.) durch und $53 \%$ informierten auf der schuleigenen Website über Neuigkeiten. $42 \%$ forderten das Kollegium auf, Schüler*innen telefonisch zu kontaktieren und diese über Neuigkeiten zu informieren und 31 \% bevorzugten für das Kommunizieren Messenger-Dienste. Dabei ordneten auch $13 \%$ der befragten Schulleitungen, das sind 8 Personen, das Kommunizieren über Social-Media-Kanäle (Instagram, Facebook etc.) an. 5 \% arbeiteten mit webbasierten Foren. Zwei Schulen nannten zusätzlich noch die Papierform.

In Abbildung 6 wird auf die Art und Weise der Vermittlung von Lern- und Unterrichtsaufgaben sowie Arbeitsaufträgen an die Schüler*innen eingegangen.

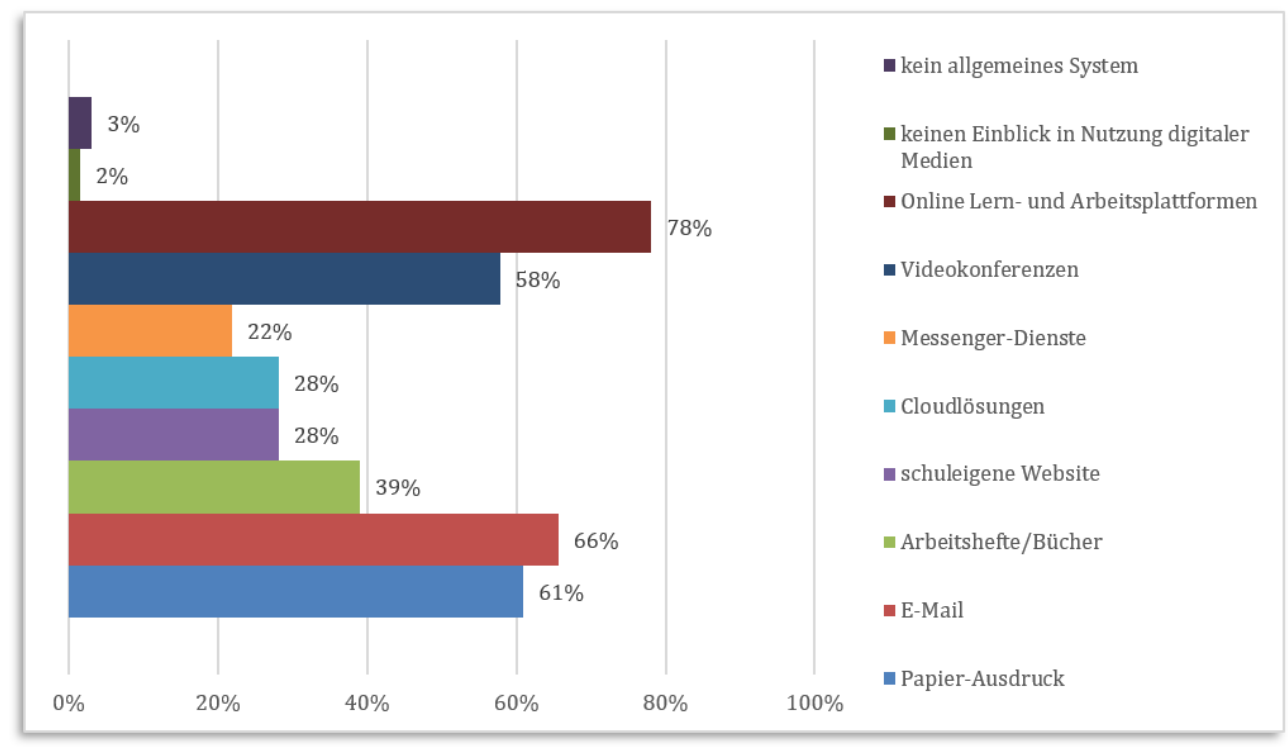

Abbildung 6: Medien für Aufgaben ( $n=64)$.

Ergebnisse der Befragung von 69 Schulleitungen an steirischen Mittelschulen im Oktober 2020.

$78 \%(n=64)$ gaben an, diese über Online-Lern- und Arbeitsplattformen übermittelt zu haben. $66 \%$ verwendeten für die Übermittlung von Arbeitsaufträgen E-Mails, 61 \% bevorzugten die Ausgabe gedruckter Materialien per Post und $58 \%$ (37 der 64 Schulen) ordneten an, Videokonferenzen mit den Schüler*innen durchzuführen. Nur $39 \%$ bestanden auf den Einsatz von Arbeitsheften und Büchern. Jeweils $28 \%$ nutzen die schuleigene Website bzw. diverse Cloud-Dienste, um einen Download von Lern- und Unterrichtsaufgaben zu ermöglichen. $22 \%$ der Schulen verwendeten Messenger-Dienste (WhatsApp etc.), um Materialien an die Lernenden zu verschicken. $3 \%$ der befragten Direktor*innen gaben an, kein allgemeines System an der Schule besessen zu haben, und eine Schulleitung gab an, keinen Einblick in die Nutzung digitaler Medien zu haben. Wurde die Kommunikation mit 
den Schüler*innen per E-Mail und über Online-Lern- und -Arbeitsplattformen angeordnet, dann erhielten die Lernenden auch ihre Lern- und Unterrichtsmaterialien sowie Arbeitsaufträge über diese Medien.

Obwohl eigentlich „nur“ 78 \% der Schulen $(n=64)$ angaben, die Lern- und Unterrichtsmaterialien über OnlineLern- und Arbeitsplattformen verteilt zu haben, antworteten auf die Fragestellung „Welche Online-Lern- und Arbeitsplattformen wurden für den Einsatz von der Direktion angeordnet?" nur $3 \%$ (2 von 64 steirischen Mittelschulen), keine Online-Lern- und Arbeitsplattformen verwendet zu haben. Aus den Antworten zu dieser Fragestellung geht hervor, dass mehr als die Hälfte der befragten Direktor*innen (56\%) mit MS Teams gearbeitet hat. 30 \% nutzten während der Schulschließung SchoolFox, 9 \% Eduvidual und 5 \% Edmodo. 41 \% verwendeten sonstige Plattformen, davon wurde insgesamt neun Mal der Anbieter Google genannt. Fünf Schulen verwendeten Google Classroom, drei Direktor*innen ordneten den Einsatz von G Suite for Education an und eine Schule arbeitete mit Google Drive. EduPage wurde von acht Schulen eingesetzt, LMS und edhu von jeweils drei. Zusätzlich wurden jeweils einmal Dropbox, Padlet, Webex und ein selbsterstellter Down- und Upload-Bereich genannt $(n=64)$.

Passend zu den eingesetzten Online-Lern- und Arbeitsplattformen wird nun erneut auf Videokonferenzen, die bereits im vorherigen Subkapitel analysiert wurden, eingegangen. Von den $56 \%$ der Schulleitungen, die darauf bestanden, dass Lehrer*innen mit ihren Schüler*innen Präsenzzeiten für digitalen Live-Unterricht vereinbarten, beantworten 32 (von 34) Personen die Frage, welches zentrale Tool dafür eingesetzt und angeordnet wurde. 53 \% verwendeten MS Teams für Videokonferenzen, zwei Schulen wendeten Zoom an und jeweils eine Schule SchoolFox bzw. WebEx. 38 \% der Teilnehmer*innen nannten andere Tools. Insgesamt wurde acht Mal der Anbieter Google genannt. Hier wird davon ausgegangen, dass Google Meet eingesetzt wurde. Außerdem wurde einmal EduPage angeführt.

Ergänzend zu den verwendeten Online-Lern- und Arbeitsplattformen wurde auch die Art der Übermittlung von Rückmeldungen erhoben, welche in Abbildung 7 veranschaulicht ist.

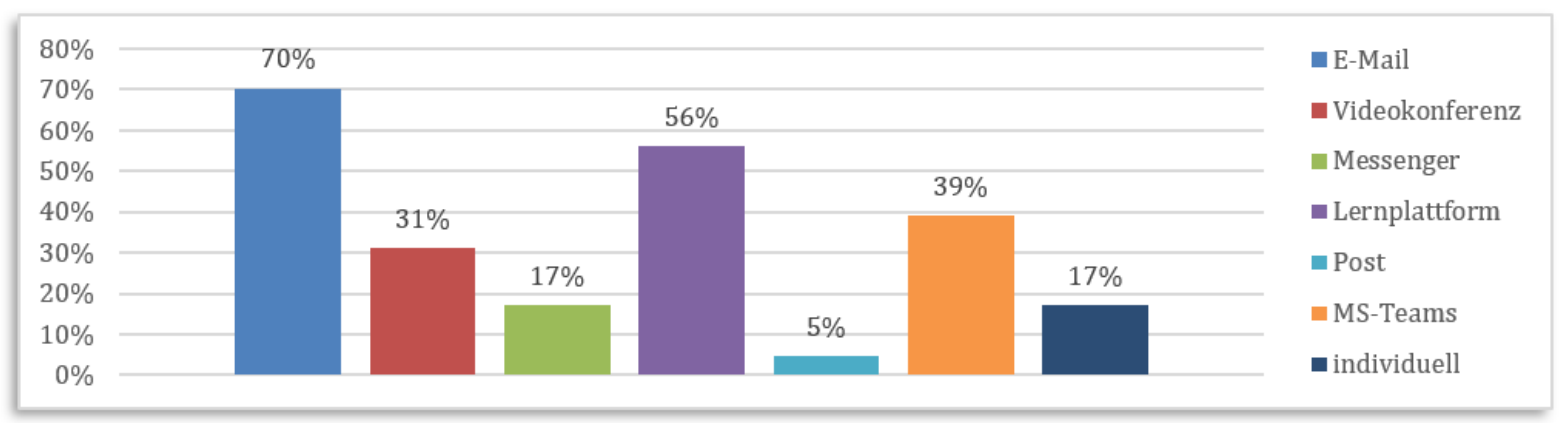

Abbildung 7: Tools für Rückmeldungen an Lernende ( $n=64)$.

Ergebnisse der Befragung von 69 Schulleitungen an steirischen Mittelschulen im Oktober 2020.

Die meisten Schulen ( $n=64)$ übermittelten Rückmeldungen per E-Mail (70\%) und über die von der Schule gewählte Lernplattform (56\%). $39 \%$ aller Befragten nannten MS Teams als Rückmeldungstool und $31 \%$ übermittelten Feedback in Videokonferenzen. $17 \%$ der Schulen verschickten Rückmeldungen mit diversen Messenger-Diensten und drei Schulen wählten für das Verschicken die Post. Außerdem nannten $17 \%$ der Schulleitungen, dass das Kollegium auf individuelle Art und Weise Rückmeldungen gab.

Nicht nur für das Übermitteln von Rückmeldungen war der E-Mail-Verkehr mit Abstand am bedeutendsten, sondern auch für das Informieren der Eltern und Erziehungsberechtigten. Wie Informationen an die Eltern und Erziehungsberechtigten übermittelt wurden, ist in Abbildung 8 dargestellt. 
R\&E-SOURCE https://journal.ph-noe.ac.at

Online Journal for Research and Education

Ausgabe 16, Oktober 2021, ISSN: 2313-1640

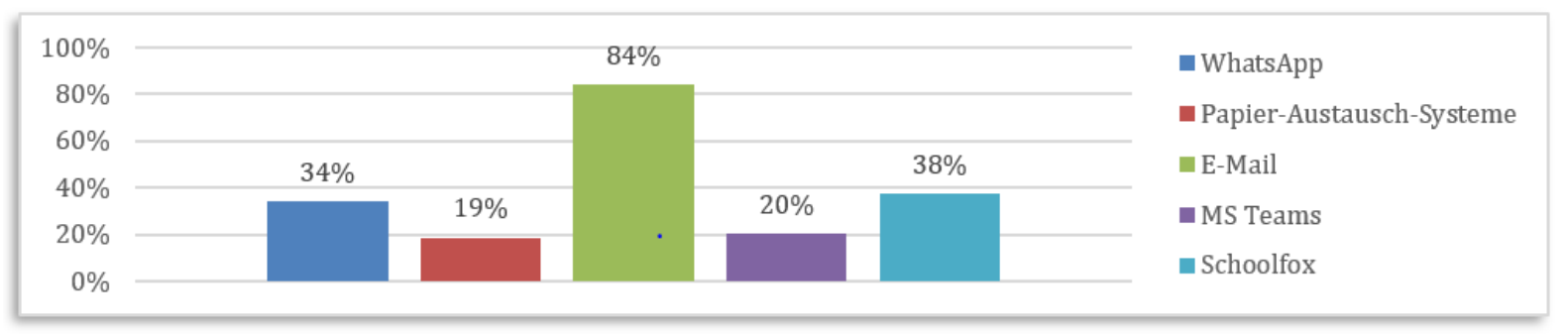

Abbildung 8: Tools für Informationsvermittlung an Eltern und Erziehungsberechtigte ( $n=64)$.

Ergebnisse der Befragung von 69 Schulleitungen an steirischen Mittelschulen im Oktober 2020.

$84 \%$ aller Schulen $(n=64)$ informierte die Eltern und Erziehungsberechtigten per E-Mail über Neuigkeiten. Im Frühjahr 2020 nutzen zudem bereits $38 \%$ der Schulen SchoolFox für die Informationsübermittlung. $34 \%$ verwendeten WhatsApp, $20 \%$ MS Teams und $19 \%$ aller Schulen setzten noch auf Papier-Austausch-Systeme wie Briefe.

Wie aus der Auswertung hervorgeht, ordneten viele Direktor*innen die Nutzung von MS Teams und SchoolFox an. Passend dazu folgt die Aufzählung der häufigsten genannten Tools hinsichtlich der offenen Fragestellung, welche konkreten Tools und Internetseiten die Mittelschulen anderen Schulen empfehlen. Es muss angemerkt werden, dass bei der Beantwortung der Fragestellung Mehrfachnennungen möglich waren. $42 \%$ antworteten mit MS Teams ( $n=36)$, ergänzend dazu nannten zwei Schulen Office 365, das MS Teams inkludiert. $28 \%$ der Schulleiter*innen empfehlen SchoolFox, $14 \%$ sind von Eduvidual überzeugt und $11 \%$ sind vom Angebot der Plattform Eduthek, auf der seitens des BMBWF übersichtlich aufbereitetes Lern- und Übungsmaterial für alle Schulstufen zur Verfügung gestellt wird, beeindruckt.

\subsection{Informationsvermittlung, Teamwork, Kenntnisse und Vorgehensweisen im Kollegium}

Im Folgenden wird zur Beantwortung der Forschungsfrage und der dazugehörigen Leitfragen die Wichtigkeit unterschiedlicher Aspekte im Zusammenhang mit dem Kollegium abgebildet und beschrieben.

Die Informationsvermittlung an das Kollegium hat an allen Schulen besondere Aufmerksamkeit erhalten. $62 \%$ der Schulleitungen $(n=65)$ kontaktierten das Kollegium regelmäßig ( $M=1 ; M D=1 ; s=0,659)$. An $29 \%$ der Schulen erfolgte die Informationsvermittlung mehrmals in der Woche und an $9 \%$ wurde eine Kommunikation mit dem Kollegium einmal in der Woche durchgeführt. An keiner der befragten Schulen fanden die Informationsvermittlungen seltener statt.

Auf gegenseitige Unterstützung wurde großer Wert gelegt.

Das Kollegium war engagiert.

Unterlagen wurden für den Unterricht gemeinsam erstellt.

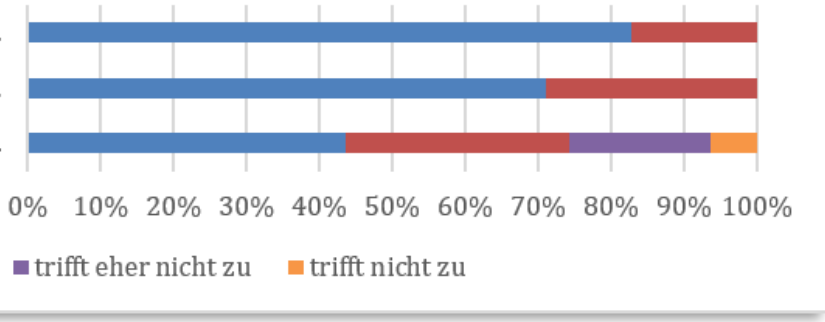

- trifft zu $\quad$ trifft eher zu $\quad$ trifft eher nicht zu $\quad$ trifft nicht zu

Abbildung 9: Zusammenhalt im Team.
Ergebnisse der Befragung von 69 Schulleitungen an steirischen Mittelschulen im Oktober 2020.

Nicht nur häufige Kontaktaufnahme zwischen der Schulleitung und dem Kollegium war während der Schulschließung von besonderer Wichtigkeit, sondern auch die gegenseitige Unterstützung im Kollegium (s. Abbildung 9). $83 \%$ der Befragten $(n=64)$ gaben an, dass an der jeweiligen Schule großer Wert auf gegenseitige Unterstützung im Kollegium gelegt wurde, $17 \%$ sind der Meinung, dass dies eher zutraf, keine Schulleitung stimmte (eher) dagegen ( $M=4 ; M D=4 ; s=0,377)$. $71 \%$ der Direktor*innen antworteten zudem, dass das Kollegium für den Einsatz digitaler Medien engagiert war, 29 \% sind der Meinung, das Kollegium war eher engagiert und keine Schulleitung stimmte dagegen ( $M=4 ; M D=4 ; s=0,454)$. 
Ergänzend zur gegenseitigen Unterstützung, die an allen Schulen gegeben war, trugen $44 \%$ der Schulleitungen ( $n=62$ ) den Kolleg*innen auf, gemeinsam Unterlagen für den digitalen Unterricht zu erstellen. $31 \%$ ordneten dies eher an, $19 \%$ der Schulen schrieben das eher nicht vor und $6 \%$ gaben das Erstellen gemeinsamer Unterlagen nicht vor ( $M=3 ; M D=3 ; s=0,935)$.

Nicht nur das Resultat der gemeinsamen Erstellung von Unterlagen, sondern auch die Ergebnisse der Einschätzung, wie kompetent das Kollegium für den Einsatz digitaler Medien war, fiel äußerst positiv aus ( $n=63$ ). Hier liegt das arithmetische Mittel bei $75 \%$ und der Median bei $80 \%$, die Standardabweichung beträgt 17,565 \%. Durchschnittlich sind die Schulleiter*innen somit der Meinung, dass 75 \% des Kollegiums für den Einsatz digitaler Medien kompetent sind.

Die regelmäßige Informationsvermittlung an das Kollegium, gegenseitige Unterstützung und das gemeinsame Erstellen von Unterlagen waren während der Schulschließung im Frühjahr 2020 besonders wichtig, um Distance Learning an den steirischen Mittelschulen zu ermöglichen. Aber auch die regelmäßige Absprache bezüglich des weiteren Vorgehens im Fachteam war äußerst bedeutend. Mehr als der Hälfte der Schulleitungen war es ein Anliegen, die Fachkolleg*innen regelmäßig miteinander in Kontakt treten zu lassen. $32 \%$ ordneten eine wöchentliche Absprache im Fachteam an und $8 \%$ plädierten, dies mehrmals in der Woche durchzuführen. Nur $5 \%$ (3 Schulen) ordneten die Absprache seltener als alle zwei Wochen an und zwei Schulen gaben diese nie vor $(n=60 ; M=2 ; M D=1 ; s=1,345)$.

\subsection{Zukünftige Rolle digitaler Medien aus Sicht der Schulleitungen und ihre Anliegen}

Das letzte Subkapitel der Untersuchungsergebnisse widmet sich der zukünftigen Rolle digitaler Medien an steirischen Mittelschulen, den Investitionen in digitale Medien und den Wünschen und Anliegen, die Schulen in Bezug auf Digitalisierung an die Regierung und an das Bildungsministerium haben. In Abbildung 10 wird die zukünftige Rolle, die digitale Medien an den Schulen einnehmen werden, veranschaulicht.

Digitale Medien werden eine bedeutende Rolle..

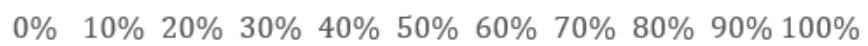

- trifft zu $\quad$ - trifft eher zu $\quad$ - trifft eher nicht zu $\quad$ a trifft nicht zu

Abbildung 10: Zukünftige Rolle digitaler Medien im Schullalltag.

Ergebnisse der Befragung von 69 Schulleitungen an steirischen Mittelschulen im Oktober 2020.

$63 \%$ der befragten Direktor*innen $(n=60)$ sind der Meinung, dass digitale Medien an ihrer Schule in Zukunft eine sehr bedeutende Rolle spielen und viel häufiger im Unterricht eingesetzt werden als bisher $(M=4 ; M D=4$; $s=0,669)$. $30 \%$ der Befragten gaben an, diese werden in Zukunft eine eher wichtige Rolle spielen. Insgesamt sind nur vier Schulleitungen der Meinung, an ihrer Schule werden digitale Medien in Zukunft (eher) keine tragende Rolle einnehmen und (eher) nicht häufiger im Unterricht eingesetzt als bisher.

Passend zur zukünftigen Wichtigkeit digitaler Medien wurde untersucht, ob die Schule/die Gemeinde in diesem Schuljahr (2020/21) mehr Geld in digitale Medien und Geräte investieren wird als in den letzten Jahren. Ein Drittel der steirischen Mittelschulen wird mit Sicherheit mehr Geld investieren. Es kann davon ausgegangen werden, dass sich diese Schuleiter*innen in Zukunft für häufigeres Einbinden digitaler Medien im Unterricht einsetzen werden. 17 \% sprachen sich trotz der COVID-19-Krise gegen eine größere Investition in digitale Medien aus. Aus der Untersuchung können keine Begründungen entnommen werden, es geht nicht hervor, ob diese 17 \% bereits über eine moderne digitale Ausstattung verfügen oder nicht, dennoch wird davon ausgegangen.

In Hinblick auf die Zukunft beschrieben 48 Schulleitungen ihre Wünsche und Anliegen, die sie in Bezug auf Digitalisierung an die Regierung und an das Bildungsministerium haben. Bei dieser Fragestellung bestand die Möglichkeit, pro Schule mehrere Aspekte zu äußern. Genau zwei Drittel der Schulen wünschen sich im Gesamten eine bessere technische Ausstattung, um Digitalisierung an Schulen zu ermöglichen und voranzutreiben. Die Finanzierung und Bereitstellung von Endgeräten für Schüler*innen wie auch für das Lehrpersonal und eine flächendeckende Versorgung mit Breitband sind dafür unumgänglich. Die Schulerhalter*innen sollen zudem eine finanzielle Unterstützung für eine gut funktionierende Infrastruktur erhalten, den Gemeinden fehlt die 
Zuwendung seitens des Landes. $27 \%$ kritisieren den Zugang zu den unterschiedlichen Lizenzen und die Unterschiede zwischen Landes- und Bundesschulen. Allen Schüler*innen sollen an allen Schulen dieselben Möglichkeiten geboten werden. $4 \%$ der Befragten fordern außerdem ein erhöhtes Stundenkontingent für digitalen Unterricht. Mit der bisherigen Stundenanzahl und der Gesamtschülerzahl von 25 in einer Klasse kann laut Umfrage kein gewinnbringender Unterricht durchgeführt werden.

\section{Diskussion der Ergebnisse}

Mit der vorliegenden Umfrage und ihren Ergebnissen können detaillierte Einblicke in die Situation an steirischen Mittelschulen vor und während der Schulschließungen in der ersten COVID-19-Welle von März bis Juni 2020 gegeben werden. Die Daten wurden dabei retrospektiv erhoben und es ist anzunehmen, dass eher Schulleitungen den Fragebogen ausfüllten, die diese Situation vergleichsweise gut bewältigt haben - beides könnte dazu führen, dass es kleinere Abweichungen von der tatsächlichen Situation bzw. Verzerrungen der Darstellungen gibt. Bei der Befragung selbst gab es bei manchen Fragen auffallende Werte, die wir bereits bei der Ergebnisdarstellung diskutiert haben. Dass wir die Befragung anonym durchgeführt haben, hat uns leider die Möglichkeit genommen, an einigen Stellen nachzufragen - sollte aber dazu führen, dass wir auch eher authentische Beschreibungen bekommen. Zur Einordnung der Ergebnisse ist zu ergänzen, dass für steirische Mittelschulen schon bereits vor der COVID-19-Pandemie etliche Grundlagen gelegt wurden, die die Arbeit mit digitalen Fernunterrichtsformen erleichterten, u. a. durch die Initiativen zur digitalen Grundbildung (BMBWF, 2018b), den Masterplan für Digitalisierung (BMWBF, 2018a) oder auch den 8-Punkte-Plan für einen digitalen Unterricht (BMBWF, 2020f).

Im Vergleich zu Studien, die im Vergleichszeitraum zur Situation des Unterrichts und des Technologieeinsatzes während der Schulschließungen im deutschsprachigen Raum entstanden sind, lassen sich zudem folgende Übereinstimmungen und Abweichungen feststellen:

Eickelmann und Drossel (2020) stellten für deutsche Schulen fest, dass diese nicht über Konzepte verfügten, die eine Versorgung der Schüler*innen mit Lernangeboten für die Zeit einer Schulschließung sicherstellt (S. 12f). Bei den befragten steirischen Mittelschulen gaben vergleichsweise viele, nämlich rund ein Fünftel, an, dass sie über ein solches Konzept verfügten. Inwieweit sich diese Vorerfahrungen bzw. Unterschiede in der Umsetzung vor der Pandemie der von uns befragten Mittelschulen auf ihre Gestaltung während der ersten Distanzunterrichtsphase ausgewirkt haben, bedarf noch detaillierter Auswertung.

Mehrere unterschiedliche Angaben gibt es zur Rolle des Live-Unterrichts während des Distance Learnings mit Hilfe von Videokonferenzsystemen in den Schulen im deutschsprachigen Europa: Nach Huber et al. (2020) wurde digitaler Live-Unterricht an den österreichischen, schweizerischen und deutschen Schulen im Frühjahr 2020 eher selten eingesetzt (S. 26), 58 \% der schulischen Mitarbeiter*innen und die Hälfte der Schulleitungen äußerten, dass es keine Vereinbarung für digitalen Live-Unterricht gab (Huber et al., 2020, S. 56). Der Untersuchung „So sehen Lehrkräfte die Corona-Krise in der Bildung" zufolge hielten nur 35,5\% der befragten Lehrer*innen in Deutschland Videokonferenzen ab (So sehen Lehrkräfte die Corona-Krise in der Bildung, 2020, Abschn. Digitaler Fernunterricht-wie kommunizieren Lehrkräfte?). Die österreichische Studie „Lernen trotz Corona“ kam hingegen zu dem Ergebnis, dass diese Unterrichtsform von mehr als der Hälfte der Befragten eingesetzt wurde (Tengler et al., 2020, S. 20). Ein genauerer Blick auf Schulform, Fragezeitpunkt, Befragte und Fragestellungen könnte diese Unterschiede vielleicht zu Teilen erklären, vermutlich wurden jedoch in Österreich Videokonferenzsysteme in einem weitaus höheren Umfang eingesetzt. In jedem Fall zeigt die hier vorgestellte Befragung, dass mehr als die Hälfte der Schulleitungen der befragten steirischen Mittelschulen darauf bestand, dass digitaler Live-Unterricht angeboten wurde, viele davon kontrollierten diese Anweisung.

Laut der vorgelegten Befragung haben die Schulleitungen an steirischen Mittelschulen innerschulische Online-Fortbildungen angeboten, dennoch gab fast die Hälfte der Schulleitungen an, dass sich die Kolleg*innen Kompetenzen auch in Eigenregie aneigneten. Hier zeigt die österreichische Befragung von Tengler et al. (2020, S. 28), dass sich mehr als die Hälfte der Lehrer*innen Dinge auch im Selbststudium oder durch Weiterbildungsteilnahme angeeignet hat. Es ist zu vermuten, dass dies auch für Lehrkräfte an den steirischen Mittelschulen gilt.

Nach der Durchführung und Auswertung der Befragung wurde eine weitere Befragung von Schulleitungen in Österreich publiziert, die Vergleiche ermöglicht: Jesacher-Rößler und Klein (2020) befragten bereits im Juni und Juli 2020532 Schulleitungen von allgemeinbildenden und berufsbildenden öffentlichen Schulen in Österreich. Sie stellen dabei fest, dass Strategien im Umgang mit dem Distanzunterricht an Neuen Mittelschulen „deutlich häufiger schul-, fach- oder jahrgangsweit abgestimmt wurden als an den anderen Schulformen“ (S. 5). Die 
Erhebung erfolgte wiederum in einem internationalen Kontext (Bremm et al., 2021), sodass nun auch weitere Vergleiche möglich sind. So wird dabei festgestellt, dass die Diskussion der Lernziele und -erwartungen, die in Österreich nach den Osterferien erfolgte - werden neue Inhalte unterrichtet, wie wird vermieden, dass Kinder abgehängt werden -, in Deutschland und der Schweiz nicht akzentuiert wurde (S. 133). Unterschiede zeigen sich so z. B. auch in der Unterstützung der Schulleitungen durch das Ministerium und zuständige Stellen: So gaben nur „7.7 Prozent der Zürcher Schulleitungen an, zu wenig Informationen erhalten zu haben, während dies in Österreich auf 29.0 Prozent und in NRW auf 59.4 Prozent der Befragten zutraf" (S. 132f). Die technische Ausstattung und Unterstützung der Schulen werden dabei im Beitrag insgesamt in der Schweiz positiver und in Deutschland schlechter beschrieben als in Österreich. Hier könnten zukünftig auch Vergleiche von Infrastrukturen und Kompetenzen weitere Einblicke geben (vgl. Bock-Schappelwein et al., 2021).

Nicht alles, was von Seiten der Schulleitungen organisiert, geplant, vorgegeben und unterstützt wurde, ist vermutlich unmittelbar so erfolgt und umgesetzt worden. Nicht alles kam so auf Seiten der Lehrer*innen, der Schüler*innen oder auch der Eltern, die in den Zeiten der Schulschließung nicht nur intensiver bei der Betreuung, sondern teils auch beim Unterricht und Lernen gefordert und eingebunden waren, an. Beiträge zur Situation während der Schulschließungen in Österreich zeigen so die großen Herausforderungen und Unterschiede, zum Beispiel für Schüler*innen und Eltern (z. B. Kämpf und Winetzhammer, 2020; Pelikan et al., 2021) sowie für Lehrer*innen (Reischl und Schmölz, 2020). Die unterschiedlichen Perspektiven und Erfahrungen sind hier zukünftig ggf. auch abzugleichen und weitergehend zu untersuchen.

\section{Ausblick}

Die Forschungsergebnisse führen zu der Schlussfolgerung, dass das Distance Learning an den steirischen Mittelschulen zahlreiche Digitalisierungsinitiativen in Schwung gebracht hat: Der Wunsch nach verstärkter Digitalisierung spiegelt sich auch in der Bereitschaft der Schulleitungen der steirischen Mittelschulen wider, mehr in diesen Bereich zu investieren. Im Schuljahr 2020/21, so die Ergebnisse der Befragung, solle auch mehr Geld in digitale Medien und Geräte fließen als in den letzten Schuljahren. Die COVID-19-Krise kann so für die Mittelschulen als Treiber und Chance im Hinblick auf Digitalisierung interpretiert werden. So gesehen haben sich der enorme Arbeitseinsatz der Schulleiter*innen sowie die mit viel Zeitaufwand und Unsicherheiten verbundene Bereitschaft der Lehrer*innen, Unterricht für das Distance Learning völlig neu zu denken und zu gestalten, gelohnt. Zukünftige Lernprozesse sollten verstärkt Differenzierungs- und Individualisierungsangebote beinhalten, ebenso muss der Weg der Schulen von der Bildungsdirektion sowie dem BMBWF begleitet und unterstützet werden, um den durch die COVID-19-Pandemie entstandenen Lücken und Unterschieden der Wissens- und Kompetenzstände der Schüler*innen gerecht zu werden. Die Befragung zeigt zudem auch, dass es bei einigen der 2020 genutzten Tools Datenschutzbedenken gibt - ein Achtel der befragten Schulleiter*innen gab an, dass Social Media als Kommunikationsmittel für den Austausch mit Schüler*innen genutzt wurde - und es hier trotz der gewonnenen Kompetenzen auch noch Raum für Anpassungen gibt.

\section{Literatur}

BMBWF (2018a). Masterplan Digitalisierung. Vortrag an den Ministerrat, BMBWF-36.200/0023Präs/C/PM/2018. https://www.bmbwf.gv.at/dam/jcr:775c5fc4-6dad-41b7-bb91bb0f8a215a43/masterplan_digitalisierung_mrv.pdf

BMBWF (2018b). Verordnung der Bundesministerin für Bildung, mit der die Verordnung über die Lehrpläne der Neuen Mittelschulen sowie die Verordnung über die Lehrpläne der allgemeinbildenden höheren Schulen geändert werden. Bundesgesetzblatt Nr. II Nr. 71/2018. https://www.ris.bka.gv.at/eli/bgbl/Il/2018/71/20180419

BMBWF (2020a, März 12). Umgang des Bildungssystems mit dem Coronavirus-Eckpunkte. https://www.bmbwf.gv.at/Themen/schule/beratung/corona/corona_info.html

BMBWF (2020b, März 13). Umgang des Bildungssystems mit dem Coronavirus-Erlass-Teil 2. https://www.bmbwf.gv.at/Themen/schule/beratung/corona/corona_info.html

BMBWF (2020c, März 31). Leitlinien für die Fernlehre/das Distance Learning nach den Osterferien. https://www.bmbwf.gv.at/Themen/schule/beratung/corona/corona_info.html 
BMBWF (2020d). Eckpunkte der Aktivierung des Schulsystems.

https://www.bmbwf.gv.at/Themen/schule/beratung/corona/corona_info/corona_etappenplan.html

BMBWF (2020e). Umsetzung des Etappenplans für Schulen: Richtlinien für die Unterrichtsorganisation und die pädagogische Gestaltung.

https://www.bmbwf.gv.at/Themen/schule/beratung/corona/corona_info/corona_etappenplan.html

BMBWF (2020f). 8-Punkteplan. https://digitaleschule.gv.at/\#8punkteplan

Bock-Schappelwein, J.; Firgo, M.; Kügler, A. \& Schmidt-Padickakudy, N. (2021). Digitalisierung in Österreich: Fortschritt, digitale Skills und Infrastrukturausstattung in Zeiten von COVID-19, WIFO Monatsberichte 6/2021, S. 451-459.

https://www.wifo.ac.at/jart/prj3/wifo/resources/person_dokument/person_dokument.jart?publikati onsid=67254\&mime_type=application/pdf

Brandhofer, G., Buchner, J., Höfler, E., Schrammel, N., \& Tengler, K. (2020). Lernen trotz Corona.

Medienimpulse, 58(2), 13. https://doi.org/10.21243/MI-02-20-33

Bremm, N.; Jesacher-Rößler, L.; Klein, E. D.; Racherbäumer, K. (2021). Covid 19 - Herausforderungen und Chancen für die Schulentwicklung. Ausgewählte Ergebnisse einer international vergleichenden Studie zum Schulleitungshandeln in Deutschland, Österreich und der Schweiz. In: C. Reintjes, R. Porsch \& G. Im Brahm, Grit (Hrsg.), Das Bildungssystem in Zeiten der Krise. Empirische Befunde, Konsequenzen und Potenziale für das Lehren und Lernen. Münster: Waxmann, S. 117-136.

Eickelmann, B. (2020). Wie der Blick in die Zahl für den Blick nach vorne hilft: Perspektiven aus der Studie ICILS 2018 in der Zeit der Corona-Krise und darüber hinaus. 46(4), 6-10.

Eickelmann, B., \& Drossel, K. (2020). Schule auf Distanz: Perspektiven und Empfehlungen für den neuen Schulalltag. Eine repräsentative Befragung von Lehrkräften in Deutschland. Vodafone Stiftung Deutschland. https://www.vodafone-stiftung.de/wp-content/uploads/2020/05/Vodafone-StiftungDeutschland_Studie_Schule_auf_Distanz.pdf

Faßmann, H. (2020, März 12). Umgang des Bildungssystems mit dem Coronavirus-Erlass. https://www.bmbwf.gv.at/Themen/schule/beratung/corona/corona_info.html

Faßmann, H., Gangl, R., Kimberger, P., \& Weiss, H. (2020, Mai 1). Schulautonome Tage. https://www.bmbwf.gv.at/Themen/schule/beratung/corona/corona_info.html

Gerhardts, L., Kamin, A.-M., Meister, D. M., Richter, L., \& Teichert, J. (2020). Entwicklung von Selbstlern- und Medienkompetenz im Homeschooling - Chancen und konzeptionelle Anforderungen. Zeitschrift für Schul- und Professionsentwicklung, 2(6), 129-154.

Huber, S. G., Günther, P. S., Schneider, N., Helm, C., Schwander, M., Schneider, J. A., \& Pruitt, J. (2020). COVID19 - aktuelle Herausforderungen in Schule und Bildung: Erste Befunde des Schul-Barometers in Deutschland, Österreich und der Schweiz. Münster: Waxmann.

https://www.waxmann.com/index.php?elD=download\&buchnr=4216

Jesacher-Rößler, L. \& Klein, E. D. (2020). COVID-19: Strategien der Schulentwicklung in der Krise. Ergebnisse einer Schulleitungsbefragung in Österreich. Working Paper,Innsbruck: Arbeitsbereich Schulentwicklungsforschung und Leadership, Institut für LehrerInnenbildung und Schulforschung,Universität Innsbruck. DOI: 10.25651/1.2020.0010.

Kämpf, P., \& Winetzhammer, A. (2020). Distance Learning während der Corona-Krise an Österreichs Volksschulen. Medienimpulse, 58(02), https://doi.org/10.21243/mi-02-20-29

Langner, A. (2020). Schule ohne Schule - Perspektiven von Eltern und Lernbegleiter*innen. Zeitschrift für Schulund Professionsentwicklung, 2(6), 100-122.

Pelikan, E.R., Lüftenegger, M., Holzer, J. et al. (2021). Learning during COVID-19: the role of self-regulated learning, motivation, and procrastination for perceived competence. In: Zeitschrift für Erziehungswissenschaft, 24, 393-418. https://doi.org/10.1007/s11618-021-01002-x

Reischl, J., \& Schmölz, A. (2020). „Ich bin keine Pausenaufsicht, ich bin kein Retter ... ich bin Lehrer.": Eine dokumentarische Analyse zur Rolle von Lehrenden im "home schoolings" der COVID-19-Krise. Medienimpulse, 58(02). https://doi.org/10.21243/mi-02-20-23

So sehen Lehrkräfte die Corona-Krise in der Bildung. (2020, April 13). Fobizz. https://fobizz.com/umfrageergebnisse-so-sehen-lehrkraefte-die-corona-krise-in-der-bildung/

Tengler, K., Schrammel, N., \& Brandhofer, G. (2020). Lernen trotz Corona. Chancen und Herausforderungen des distance learning an österreichischen Schulen. Medienimpulse, 58(2), 37. https://doi.org/10.21243/MI-02-20-24

Weber, H. (2021). Distance Learning an steirischen Mittelschulen in Zeiten der COVID-19-Pandemie. https://bit.ly/3kc4lvd 
R\&E-SOURCE https://journal.ph-noe.ac.at

Online Journal for Research and Education

Ausgabe 16, Oktober 2021, ISSN: 2313-1640

Wrase, M. (2020). Schulrechtliche Herausforderungen in Zeiten der Corona-Pandemie. In D. Fickermann \& B. Edelstein (Hrsg.), „Langsam vermisse ich die Schule ...". Schule während und nach der CoronaPandemie, Münster: Waxmann, S. 105-116. https://doi.org/10.31244/9783830992318.06 